\title{
Yellow Laser Stimulation at GV2 Acupoint Mitigates Apoptosis, Oxidative Stress, Inflammation, and Motor Deficit in Spinal Cord Injury Rats
}

\author{
Parichat On-ong-arj, ${ }^{1}$ Jintanaporn Wattanathorn $\left(\mathbb{D},{ }^{2,3}\right.$ \\ Supaporn Muchimapura $\mathbb{D}^{2,3}$ and Wipawee Thukham-mee ${ }^{2,3}$ \\ ${ }^{1}$ Department of Physiology (Neuroscience Program), Faculty of Medicine, Khon Kaen University, Khon Kaen, 40002, Thailand \\ ${ }^{2}$ Department of Physiology, Faculty of Medicine, Khon Kaen University, Khon Kaen, 40002, Thailand \\ ${ }^{3}$ Integrative Complementary Alternative Medicine Research and Development Center, Khon Kaen University, 40002, Thailand \\ Correspondence should be addressed to Jintanaporn Wattanathorn; jintanapornw@yahoo.com
}

Received 7 June 2018; Revised 6 September 2018; Accepted 20 September 2018; Published 8 October 2018

Academic Editor: Sakthivel Muniyan

Copyright (C) 2018 Parichat On-ong-arj et al. This is an open access article distributed under the Creative Commons Attribution License, which permits unrestricted use, distribution, and reproduction in any medium, provided the original work is properly cited.

Currently, the suppression of oxidative stress and inflammation is considered as the treatment targets of spinal cord injury due to their roles on the hindrance of recovery process. Since laser acupuncture decreased oxidative stress and enhanced the survival of neurons from oxidative stress damage and GV2 stimulation was selected as one stimulated acupoint in order to enhance the recovery of spinal cord injury, we hypothesized that laser acupuncture at GV2 should enhance the recovery of spinal cord injury. To test this hypothesis, male Wistar rats were induced spinal cord injury at T10 level and they were exposed to a 10 minute-stimulation at GV2 by yellow laser. Laser acupuncture was performed at 0.25 and 1, 2, 6, and 12 hours after spinal cord injury. Then, the stimulation was performed once daily for 7 days. Locomotor assessment was carried out on days 3 and 7 after injury. At the end of study period, the densities of polymorphonuclear of leukocyte, Bax, Caspase-3, Bcl-2, and BDNF positive stained cells in ventral horn of spinal cord were determined. Cyclooxygenase-2 (COX-2), interleukin-6 (IL-6), and oxidative stress status was also assessed. The results showed that laser acupuncture at GV2 increased BBB score, gross motor score, and densities of Bcl-2 and BDNF positive stained cells but decreased density with polymorphonuclear leukocyte, the densities of Bax and Caspase-3 positive stained cells, COX-2 level, and oxidative stress status in ventral horn of the lesion spinal cord. The reduction of serum COX-2 was also decreased. Therefore, GV2 stimulation by yellow laser might enhance the recovery of spinal cord via the increase in BDNF and the decrease in inflammation, apoptosis, and oxidative stress status in the lesion spinal cord.

\section{Introduction}

Traumatic spinal cord injury, one of the most devastating neurological disorders, produces the profound negative impacts on a patient's life and socioeconomic burdens. It has been reported that the annual Asian incidence is around 12.06 to 61.6 per million [1]. This rate is varied between developing and developed countries. However, it has been estimated that the global prevalence of spinal cord injury (SCI) each year is between 250000 and 500000 cases [2]. Despite the high impacts on socioeconomic burdens, no effective therapeutic strategy is available.
SCI consists of 2 phases of injury including the primary and secondary injuries. Primary injury occurs as the result of the compression, contusion, stretching, or kinking of the spinal cord induced by mechanical insult [3]. Following this phase, secondary injury including inflammation, oxidative stress damage, and apoptosis occurs [4]. Currently, most of the therapeutic strategies target the secondary injury because this phase plays an important role on the hindrance of recovery process following SCI.

Recent studies have demonstrated that the stimulation of the governor vessel acupoints such as GV1, GV2, and GV6 can promote the regeneration of nerve fiber at the injury site 
of spinal cord $[5,6]$ by decreasing secondary damage via the suppression of inflammation and the stimulation of nerve growth factor release [6]. In addition to the acupuncture, laser therapy is also reported to promote axonal regrowth after spinal cord injury [7] and serves as the potential strategy for neurorehabilitation for spinal cord injury [8]. Moreover, accumulative lines of evidence have demonstrated that laser can stimulate acupoint and restore numerous neuronal deficits such as brain damage and memory [9-12]. Based on the beneficial effects of laser and acupuncture together with the capability to stimulate acupoint with laser, the improvement of functional recovery of spinal cord following traumatic injury induced by laser acupuncture has gained much attention. Several studies demonstrate that the stimulation of acupoint even at single acupoint can effectively produce the desired effects [9-12]. Therefore, we hypothesized that the stimulation at GV2 acupoint which is commonly used for treating weakness or atrophy of lower limbs by yellow laser might improve apoptosis and oxidative stress and neurological deficit in SCI rats. To elucidate this issue, this study aimed to determine the effect of laser acupuncture at GV2 acupoint on locomotor activity, oxidative stress, and apoptosis in SCI rats.

\section{Materials and Methods}

2.1. Animals and Experimental Protocol. Adult male Wistar rats weighed 250-300 grams were used in this experiment. All rats were purchased from National Laboratory Animal Centre, Mahidol University, Salaya, Thailand. Rats were housed in a temperature-controlled room under a 12-hour light/dark cycle and given access to food and water ad libitum. The animals were acclimatized to the laboratory to the laboratory condition for 1 week. The protocols conducted in this study were approved by the Institutional Animal Care and Use Committee Khon Kaen University, Khon Kaen, Thailand (AEMDKKU 002/2558).

All animals were randomly divided into 5 groups as follows:

Group I: Naïve intact group: all rats in this group received no treatment.

Group II: Sham operation+ sham laser acupuncture group: all rats were subjected to sham operation surgery and received no treatment

Group III: SCI + sham laser acupuncture: rats in this group were subjected to traumatic injury at T10 level

Group IV: SCI+GV2 laser acupuncture: the experimental rats in this group were induced traumatic spinal cord injury and received GV2 stimulation induced by yellow laser.

After the induction of spinal cord injury induced by traumatic injury, rats in group III-group IV were subjected to the 10 -minute stimulation period by various interventions as described earlier. The stimulation was performed at 15 minutes, 6, 12, and 24 hours after spinal cord injury (SCI). After the first day, the 10 -minute stimulation by various interventions were performed once daily for 7 days. The neurological deficit was assessed by using a battery test comprising of Basso, Beattie, and Bresnahan (BBB) locomotor rating scale, gross motor score. These tests were performed on days 3 and 7 after SCI. At the end of study period, histomorphology of spinal cord at the lesion level was also explored by determining the densities of survival neuron, polymorphonuclear leukocytes, Bax-positve (Bax + ), caspase 3-positive (caspase $3+$ ), and $\mathrm{Bcl}-2$ positive $(\mathrm{Bcl}-2+)$ cells in ventral horn by using histology and immunohistochemistry techniques. In addition, the activity of cyclooxygenase2 (COX-2), interleukin-6 (IL-6), and oxidative stress status parameters including malondialdehyde (MDA) level, and the activities of superoxide dismutase (SOD), catalase (CAT), and glutathione peroxidase (GPx) were also investigated to probe for the possible underlying mechanism 24 hours after the first day of intervention.

2.2. Induction of Traumatic Spinal Cord Injury. After the anesthetization with Pentobarbital Sodium (50 mg/kg BW), the paravertebral muscles of experimental animals were exposed by a longitudinal incision at the midline of the back and exposed T9 to T11 vertebrae and spinal cord. The crush injury at T10 level was performed by exposing to a 15 -secondextradural compression (50 g). Following this process, muscles were closed in layers, and the incision was closed by using silk sutures no.4. The surgical wound was cared with Betadine and rats were returned to their cages with the provided food and water. The rat bladders were manually voided three times a day until they were able to regain normal bladder function. Tramadol (analgesic drug) at dose of 10/kg BW and Tetracycline (antibiotics) at dose of $50 \mathrm{mg} / \mathrm{kg} \mathrm{BW}$ were administered via subcutaneous route every 12 hours for 3 days [13].

2.3. GV2 Acupoint Stimulation. The stimulation of GV2, an acupoint located on the posterior midline and in the hiatus of the sacrum in prone position, was performed by yellow laser. The laser equipment used in this study was Weberneedle ${ }^{\circledR}$ Compact (Lauenförde, Germany) which can emit a wavelength of $589 \mathrm{~nm}$ and an output power of $50 \mathrm{~mW}$ and a diameter of the laser beam was $500 \mu \mathrm{m}$. At the end of 10 minuteintervention period, the interventions were terminated. On the first day of SCI, the interventions were applied at 15 minutes, 6, 12, and 24 hours after spinal cord injury (SCI). After the first day, the 10 minute-stimulation were performed once daily for 7 days.

\subsection{Neurological Deficit Assessments}

2.4.1. Locomotor Activity Evaluation (Basso, Beatie and Bresnahan, or BBB Score Test). The locomotion, weight support and coordination capacity following SCI of animals were assessed by using the Basso, Beattie, and Bresnahan (BBB) scale [14] was evaluated. According to this method, a score of $0-21$ was graded. The highest score or 21 represented complete mobility whereas 0 represented no spontaneous movement [15].

2.4.2. Gross Motor Score Evaluation. In addition to BBB test, the locomotor activity was also determined by assessing motor function through movement in hindlimb and weight bearing. The locomotor impairments of rats were observed 
using open field grading scores ranging from 0 (no movement in hindlimb) to 10 (normal walking). The locomotor testing was carried out for 4 minutes [16].

\subsection{Biochemical Assays}

2.5.1. Tissue Preparation and Protein Determination. After the last intervention, the lesion spinal tissue was removed and homogenized in $0.5 \mathrm{~mL}$ of ice-cold Tris- $\mathrm{HCl}$ buffer $(50 \mathrm{mM}$, $\mathrm{pH}$ 7.4). Following this process, the homogenate was subjected to a 3,000 rounds per minute (rpm)-centrifugation at $4^{\circ} \mathrm{C}$ for 15 minutes. The supernatant was harvested and stored at $-80^{\circ} \mathrm{C}$ until used. The protein concentration in the homogenate was determined by using a Thermo Scientific NanoDrop 2000c spectrophotometer (Thermo Fisher Scientific, USA and the optical density at the wavelength of $280 \mathrm{~nm}$ was determined [17].

2.5.2. Determination of Oxidative Stress Status Parameters. To assess the effect of GV2 stimulation on oxidative stress status parameters, the oxidative stress status parameters, including malondialdehyde (MDA), and the activities of superoxide dismutase (SOD), catalase (CAT), and glutathione peroxidase $(\mathrm{GPx})$ in the lesion spinal cord, were determined.

The level of MDA was determined by using thiobarbituric reaction [18]. In brief, an aliquot of sample tissue at the volume of $100 \mu \mathrm{l}$ was mixed with $100 \mu \mathrm{l}$ of $8.1 \%$ sodium dodecyl sulphate (SDS) (Sigma-Aldrich, USA), $375 \mu \mathrm{l}$ of $0.8 \%$ of thiobarbituric acid (TBA) (Sigma-Aldrich, USA), $375 \mu$ lof $20 \%$ acetic acid (Sigma-Aldrich, USA), and $150 \mu \mathrm{l}$ of distilled water (DW). Then, the mixture was boiled in a water bath at $95^{\circ} \mathrm{C}$ for 60 minutes. After cooling at room temperature, $500 \mu \mathrm{l}$ of water and $2.5 \mathrm{ml}$ of the mixture of n-butanol and pyridine at the ratio of 15:1 were added, mixed together, and centrifuged at $4,000 \mathrm{rpm}$ for 10 minutes. The supernatant was harvested and determined an absorbance of $532 \mathrm{~nm}$ by spectrophotometer. MDA level was expressed as $\mathrm{nmol} / \mathrm{mg}$ protein.

SOD activity was measured based on the ability of SOD to inhibit the reduction of cytochrome $c$ by competing for the superoxide radical. In brief, $20 \mu \mathrm{l}$ of tissue sample was added to the assay mixture containing $57 \mathrm{mM}$ phosphate buffer solution $\left(\mathrm{KH}_{2} \mathrm{PO}_{4}\right)$ (Sigma-Aldrich, USA), $0.1 \mathrm{mM}$ EDTA (Sigma-Aldrich, USA), $10 \mathrm{mM}$ cytochrome C (Sigma-Aldrich, USA) solution and $50 \mu \mathrm{M}$ of xanthine (Sigma-Aldrich, USA) solution at the volume of $200 \mu \mathrm{l}$. Then, $20 \mu \mathrm{l}$ of xanthine oxidase $(0.90 \mathrm{mU} / \mathrm{ml})$ (Sigma-Aldrich, USA) solution was added. The absorbance was measured at $415 \mathrm{~nm}$ using microplate reader. SOD enzyme (Sigma-Aldrich, USA) activities at the concentrations of $0-25$ units $/ \mathrm{ml}$ were used as standard and the results were expressed as units/mg protein [19].

Catalase activity was measured based on the rate of $\mathrm{H}_{2} \mathrm{O}_{2}$ disappearance. Briefly, $10 \mu \mathrm{l}$ of spinal cord homogenate was mixed with the assay mixture containing $50 \mu \mathrm{l}$ of $30 \mathrm{mM}$ hydrogen peroxide ((in $50 \mathrm{mM}$ phosphate buffer, $\mathrm{pH}$ 7.0) (BDH Chemicals Ltd, UK), $25 \mu \mathrm{l}$ of $\mathrm{H}_{2} \mathrm{SO}_{4}$ (SigmaAldrich, USA) and $150 \mu \mathrm{l}$ of $5 \mathrm{mM} \mathrm{KMnO} 4$ (Sigma-Aldrich, USA). Absorbance at $490 \mathrm{~nm}$ was measured using a spectrophotometer. CAT enzyme (Sigma-Aldrich, USA) at the concentration range of $0-100$ units $/ \mathrm{ml}$ was used as standard and the result was expressed as units/mg protein [20].

GPx was measured according to the procedure previously described [21]. In brief, $20 \mu \mathrm{l}$ of sample supernatant was mixed with the reaction mixture consisting of $10 \mu \mathrm{l}$ of $1 \mathrm{mM}$ dithiothreitol (DTT) (Sigma-Aldrich, USA) in $6.67 \mathrm{mM}$ potassium phosphate buffer ( $\mathrm{pH} 7$ ), $100 \mu \mathrm{l}$ of $1 \mathrm{mM}$ sodium azide (Sigma-Aldrich, USA) in $6.67 \mathrm{mM}$ potassium phosphate buffer ( $\mathrm{pH} 7$ ), $10 \mu \mathrm{l}$ of $50 \mathrm{mM}$ glutathione (SigmaAldrich, USA) solution and $100 \mu \mathrm{l}$ of $30 \%$ hydrogen peroxide (BDH Chemicals Ltd, UK). Then, the mixture was shaken for 5 minutes before adding $10 \mu \mathrm{l}$ of DTNB (5,5-dithiobis2-nitrobenzoic acid) (Sigma-Aldrich, USA). The absorbance at $412 \mathrm{~nm}$ was recorded using a spectrophotometer. The standard calibration curve was prepared by using GPx enzyme (Sigma-Aldrich, USA) at the concentration range of $0-5$ units/ml. GPx activity was expressed as units/mg protein. Then, $10 \mu \mathrm{l}$ of $10 \mathrm{mM}$ DTNB (5,5-dithiobis-2-nitrobenzoic acid) (Sigma-Aldrich, USA) was added and the optical density at $412 \mathrm{~nm}$ was recorded at $25^{\circ} \mathrm{C}$ over a period of 5 minutes. The standard calibration curve was prepared by using GPx enzyme (Sigma-Aldrich, USA) at the concentration range of $0-5$ units/ml. GPx activity was expressed as units/mg protein.

\subsubsection{Assessment of Cyclooxygenase-2 (COX-2). COX-2} activity was measured by using a commercial COX activity assay kit (Cayman Chemical, Ann Arbor, Michigan, USA). COX-2 is an enzyme that involve in inflammatory event by converting arachidonic acid to prostaglandin, an inflammatory mediator. Briefly, $10 \mu \mathrm{l}$ of tissue sample or serum, $20 \mu \mathrm{l}$ of $10 \mu \mathrm{M}$ TMPD $\left(\mathrm{N}, \mathrm{N}, \mathrm{N}^{\prime}, \mathrm{N}^{\prime}\right.$-Tetramethyl-p-phenylenediamine dihydrochloride), and $20 \mu \mathrm{l}$ of $100 \mu \mathrm{M}$ arachidonic acid were transferred into 96-well microliter plates. Following a 30 minute-incubation at room temperature, the absorbance at $590 \mathrm{~nm}$ was recorded $[22,23]$.

2.5.4. Assessment of Interleukin-6 (IL-6). The level of IL6 was determined using ELISA kit (Sigma-Aldrich, USA). The determination was performed according to the guideline protocol provided with the kit and data were expressed as $\mathrm{pg} / \mathrm{mg}$ protein. In brief, an aliquot of $100 \mu \mathrm{l}$ of tissue sample or serum was added to a 96 well-plate which coated with antibody against IL- 6 and incubated at room temperature for 150 minutes. At the end of incubation period, washing process was carried out. Then, the biotinylated antibody against IL- 6 antibody at $100 \mu \mathrm{l}$ was added and incubated at room temperature for 60 minutes. After washing and draining the solution, HRP-Streptavidin solution at $100 \mu \mathrm{l}$ was added and incubated for 45 minutes. The washing process was carried out following the incubation. Then, an ELISA Colorimetric TMB Reagent (Item H) at volume of $100 \mu \mathrm{l}$ was added and incubated at room temperature for 30 minutes. At the end of incubation period, a stop solution (Item I) at $50 \mu \mathrm{l}$ was added and the absorbance at $450 \mathrm{~nm}$ was measured. Data were presented as $\mathrm{pg} / \mathrm{ml}$.

2.6. Histological Study. Rats were sacrificed and subjected to a transcardial perfusion with $0.9 \%$ sterile saline for 5 minutes. 
TABLE 1: Effect of yellow laser acupuncture on the BBB score in rats induced spinal cord injury.

\begin{tabular}{lcrr}
\hline Treatment groups & Baseline & Day 3 & Day 7 \\
\hline Naïve intact & $21.00 \pm 0.000$ & $21.00 \pm 0.000$ & $21.00 \pm 0.000$ \\
Sham operation+ sham laser acupuncture & $21.00 \pm 0.000$ & $21.00 \pm 0.000$ & $21.00 \pm 0.000$ \\
SCI + sham laser acupuncture & $21.00 \pm 0.000$ & $0.00 \pm 0.000 * * *$,aaa & $0.00 \pm 0.000 * *$, aa \\
SCI+GV2 laser acupuncture & $21.00 \pm 0.000$ & $0.67 \pm 0.333$ & $9.17 \pm 0.167 \# \# \#$ \\
\hline
\end{tabular}

Data are expressed as mean \pm SEM and ${ }^{* *, * * *} \mathrm{P}$-value $<.01$ and .001 , respectively; compared with naïve intact, ${ }^{\text {aa,aaa }} \mathrm{P}$-value $<.01$ and .001 , respectively; compared with sham operation+ sham laser acupuncture, ${ }^{\# \#} \mathrm{P}$-value $<.001$, respectively; compared with $\mathrm{SCI}+$ sham laser acupuncture.

The spinal cord was removed and placed in $4 \%$ paraformaldehyde overnight. Following this process, the tissue was cryoprotected by incubating in $30 \%$ sucrose for 72 hours at $4^{\circ} \mathrm{C}$. Serial cross sections of the lesion spinal cord were cut at $6 \mu \mathrm{m}$ thick by using a cryostat (Thermo Scientific ${ }^{\mathrm{TM}}$ HM 525 Cryostat). Then, hematoxylin and eosin (H\&E) stain was performed. Briefly, the sections were stained with hematoxylin (Sigma-Aldrich, USA) 10 minutes and washed with water 1 minute ( 2 times). Then, the slides were immersed in $50 \%$ and $70 \%$ ethanol for 3 minutes each and stained with eosin (Sigma-Aldrich, USA) for 1 minute and dehydrated through 70, 95, 100\% ethanol for 2 minutes each. The sections were cleared with xylene for 5 minutes ( 2 times) and mounted using DPX mountant (Merck, Germany) [24]. The photographs of sections were taken using a microscope (Axio Imager.A1, Carl Zeiss, Oberkochen, Germany) equipped with a digital camera system (Axio Cam MRc 5, Carl Zeiss). The total number of polymorphonuclear leukocyte (neutrophil) per section was counted in three randomly selected fields from the lesion site per sample at 40x magnification [25].

To assess the surviving neurons density, Nissl stain was performed. The spinal cord tissue sections were immersed in $0.2 \%$ cresyl violet (Sigma-Aldrich, USA) for 2 minutes, rinsed with double distilled water, dehydrated in ethanol solutions with increasing concentrations (70, 95, 100\% 2x). Then, the sections were mounted with DPX mountant and observed under a light microscope. Normal neurons were characterized by blue staining Nissl bodies [26]. Counts were performed in three adjacent fields and expressed as the mean number of Nissl positive cells $/ 255 \mu \mathrm{m}^{2}$.

The determination of densities of Bax-positive (Bax +$)$, Caspase 3-positive (Caspase 3+), Bcl-2-positive (Bcl-2+), and brain derived nerve growth factor positive $(\mathrm{BDNF}+)$ cells were performed by using immunohistochemistry technique. In brief, spinal cord was removed and placed in $4 \%$ paraformaldehyde overnight. Then, the tissues were fixed in $30 \%$ sucrose as a cryoprotectant for 72 hour at $4^{\circ} \mathrm{C}$. The spinal cord tissues were then frozen and sectioned at $20 \mu \mathrm{m}$ thick by using cryostat. The consecutive sections were collected into six-well plates containing $0.1 \mathrm{M}$ phosphate buffered saline ( $\mathrm{pH} 7.4)$ and heated with microwave (800 watt) for 10 minutes. After heating, the sections were cooled at room temperature. Then, the sections were washed in PBS 3 times, 5 minutes each. Following this process, the sections were incubated in $0.3 \%$ hydrogen peroxide for 20 minutes. After the incubation, the sections were washed in PBS 3 times, 5 minutes each again. Following this step, the sections were blocked nonspecific binding antibodies by incubating the sections in a mixture containing $0.3 \%$ Triton X-100 (Fluka Chemika, Buchs, Switzerland), 1\% (w/v) bovine serum album (BSA) and 10\% normal goat serum for 20 minutes at room temperature. After rinsing 3 times with PBS, the sections were incubated with one of the following primary antibodies including primary antibody against Bcl-2 (1:500, Abcam, Cambridge, MA, USA), Bax (1:500, Abcam, Cambridge, MA, USA), Caspase 3 (1:500; Cell Signal Technology, Danvers, MA) and BDNF (1:500, Sigma Aldrich, Saint Louis, USA) at $4^{\circ} \mathrm{C}$ overnight. After washing in PBS, primary antibodies were detected with the REAL ${ }^{\mathrm{TM}}$ EnVision $^{\mathrm{TM}}$ Detection System, Peroxidase/DAB+ rabbit/mouse, (Dako, Glostrup, Denmark) by a 30 minute-incubation period for 30 minutes at room temperature. The sections were rinsed with PBS and incubated for 5 minutes with $3,3^{\prime}$-diaminobenzidine tetrahydrochloride (DAB) (Sigma-Aldrich, USA). Positive staining was recognized as a brown color. The control sections were stained with secondary antibody and no immunoreactive neuron was detected. The sections were mounted on gelatin-coated slides and counterstained with cresyl violet and dehydrated with graded alcohols, cleared with xylene and mounted with DPX mountant and observed under a light microscope. Immunoreactive neurons were characterized by brown granules in the cytoplasm. Counts were performed in three adjacent fields and expressed as the mean number of positive cells $/ 255 \mu \mathrm{m}^{2}[27,28]$.

2.7. Statistical Analysis. Data were expressed as mean \pm SEM. The significance of differences among the groups was assessed by using one way analysis of variance (ANOVA) test followed by Tukey test. The significant change was regarded when Pvalue $<0.05$.

\section{Results}

3.1. Effect of GV2 Stimulation on Neurological Deficits. Table 1 showed the effect of GV2 stimulation on BBB score of the animals on days 3 and 7 after the operation. When compared to naïve intact group, sham operation group showed no significant BBB score throughout the study period. SCI rats with sham laser stimulation at GV2 acupoint significantly decreased $\mathrm{BBB}$ score than sham operation group throughout the study period ( $\mathrm{P}$-value $<.01$ all; compared with sham operation group). GV2 laser acupuncture could improve BBB score at 7 days after SCI (P-value<.01; compared with SCI rats+ sham laser). The effects of GV2 laser acupuncture on gross motor score in SCI rats also showed similar pattern as BBB score as shown in Table 2. SCI rats with sham laser 
TABLE 2: Effect of yellow laser acupuncture on the gross motor score in rats induced spinal cord injury.

\begin{tabular}{lcrr}
\hline Group & Baseline & Day 3 & Day 7 \\
\hline Naïve intact & $10.00 \pm 0.000$ & $10.00 \pm 0.000$ & $10.00 \pm 0.000$ \\
Sham operation+ sham laser acupuncture & $10.00 \pm 0.000$ & $10.00 \pm 0.000$ & $10.00 \pm 0.000$ \\
SCI + sham laser acupuncture & $10.00 \pm 0.000$ & $0.00 \pm 0.000^{* * * \text {,aaa }}$ & $0.00 \pm 0.000^{* * * \text {,aaa }}$ \\
SCI+GV2 laser acupuncture & $10.00 \pm 0.000$ & $1.00 \pm 0.447$ & $3.67 \pm 0.61^{\# \# \#}$ \\
\hline
\end{tabular}

Data are expressed as mean $\pm \mathrm{SEM},{ }^{* * *} \mathrm{P}$-value $<.001$ compared with naïve intact, ${ }^{\text {aaa }} \mathrm{P}$-value $<.001$ compared with sham operation+ sham laser acupuncture, and ${ }^{\# \# \#} \mathrm{P}$-value $<.001$ compared with SCI + sham laser acupuncture.

TABLE 3: Effect of laser acupuncture at GV2 on the alterations of cyclooxygenase-2 (COX-2) and interleukin-6 (IL-6) levels in spinal cord and serum.

\begin{tabular}{lcccc}
\hline \multirow{2}{*}{ Treatment } & \multicolumn{2}{c}{ Spinal cord } & \multicolumn{2}{c}{ Serum } \\
& COX-2 $(\mathrm{ng} / \mathrm{dL})$ & IL-6 $(\mathrm{pg} / \mathrm{mL})$ & COX-2 (ng/dL) & IL-6 (pg/mL) \\
\hline Naïve intact & $17.94 \pm 0.54$ & $0.41 \pm 0.08$ & $17.24 \pm 0.89$ & $0.05 \pm 0.31$ \\
Sham operation + sham acupuncture & $18.75 \pm 1.53$ & $0.41 \pm 0.11$ & $18.59 \pm 1.63$ & $0.05 \pm 0.02$ \\
SCI+sham acupuncture & $28.64 \pm 1.50^{* * * \text {,aa }}$ & $1.60 \pm 0.60^{* * \text {,a }}$ & $27.40 \pm 1.35^{* * * \text {,aa }}$ & $0.21 \pm 0.01^{* * \text {,a }}$ \\
SCI+laser acupuncture GV2 & $19.96 \pm 1.25^{\# \#}$ & $0.65 \pm 0.16$ & $19.91 \pm 1.20^{\# \#}$ & $0.11 \pm 0.01$ \\
\hline
\end{tabular}

Data are expressed as mean $\pm \mathrm{SEM},{ }^{* *, * * *} \mathrm{P}$-value $<.01$ and .001 respectively; compared with naïve intact, ${ }^{\text {aa,aaa }} \mathrm{P}$-value $<.01$ and .001 , respectively; compared with sham operation+ sham laser acupuncture, ${ }^{\# \#} \mathrm{P}$-value $<.01$; compared with $\mathrm{SCI}+$ sham laser acupuncture.

stimulation also showed the decreased gross motor score (Pvalue<.001; compared to sham operation). The stimulation at GV2 by laser significantly enhanced gross motor score (Pvalue<.001; compared to SCI rats+ sham laser).

3.2. Effects of GV2 Stimulation on Inflammation. In this study, IL- 6 and COX-2 activity were used as indicators reflecting inflammation. The effects of GV2 stimulation on IL-6 and COX-2 in the lesion spinal cord were assessed and data were shown in Table 3. Sham operation failed to produce the significant change on both parameters just mentioned in both spinal cord and serum. SCI rats significantly increased both parameters (P-value $<.01$ and .001, respectively; compared with sham operation group) in spinal cord and serum. The stimulation of GV2 acupoint by yellow laser failed to produce significant change of IL- 6 in spinal cord and serum. Interestingly, the stimulation of $\mathrm{GV} 2$ by yellow laser significantly attenuated the elevation COX-2 in both spinal cord and serum of SCI rats (P-value $<.01$ all; compared with SCI rats+sham laser group).

3.3. Effects of GV2 Stimulation on Oxidative Stress Status. Table 4 showed the effect of GV2 stimulation on oxidative stress parameters. It was found that sham operation produced no significant changes in MDA level and the activities of SOD, CAT, and GPx in spinal cord. SCI rats which received sham laser significantly increased MDA level but decreased SOD and GPx activities (P-value<.01; P-value $<.001$ and Pvalue $<.001$, respectively; compared with sham operation group). Interestingly, GV2 laser acupuncture could decrease MDA level but increased SOD activity in the lesion spinal cord (P-value $<.01$ and $\mathrm{P}$-value $<.001$ respectively; compared with SCI rats+sham laser group).

3.4. Histological Study. Figure 1 showed the neuron density in ventral horn of spinal cord. SCI rats which received sham laser significantly decreased the density of survival neurons (P-value<.001; compared to sham operation rats). The stimulation of GV2 by laser acupuncture significantly increased neuron density in ventral horn (P-value<.001 respectively; compared with SCI rats+sham laser group). In addition, the data obtained from this study also showed that SCI rats which received sham operation significantly increased polymorphonuclear density in ventral horn of spinal cord (Pvalue <.001; compared to sham operation rats). This elevation was mitigated by the stimulation of GV2 acupoint by yellow laser (P-value<.001; compared with SCI rats+sham laser group) as shown in Figure 2.

The effect of GV2 stimulation on apoptosis in ventral horn was also explored and results were shown in Figures 3-6. SCI rats which received sham laser acupuncture significantly increased Bax and Caspase 3 but decreased Bcl-2 and BDNF positive cells densities in ventral horn (P-values $<.001$ all; compared to sham operation). When compare to naïve control, no significant changes of all parameters just mentioned were observed in ventral horn of sham operated rats. However, the increase in Bax and Caspase 3 in ventral horn of SCI rats were attenuated by GV2 laser acupuncture (P-value<.001all; compared with sham operation group). In addition, the reduction in densities of $\mathrm{Bcl}-2$ and $\mathrm{BDNF}$ positive cells was also mitigated by GV2 laser acupuncture (Pvalue $<.001$ all; compared with SCI rats+sham laser group).

\section{Discussion}

The current study has demonstrated that the stimulation of GV2 acupoint by yellow laser can enhance the structural lesion and functional recovery in spinal cord following traumatic cord injury. Locomotor activity of SCI rats which received GV2 laser acupuncture was improved. The decrease in COX-2, oxidative stress status, polymorphonuclear density, and apoptosis but increase in BDNF-positive cell density 


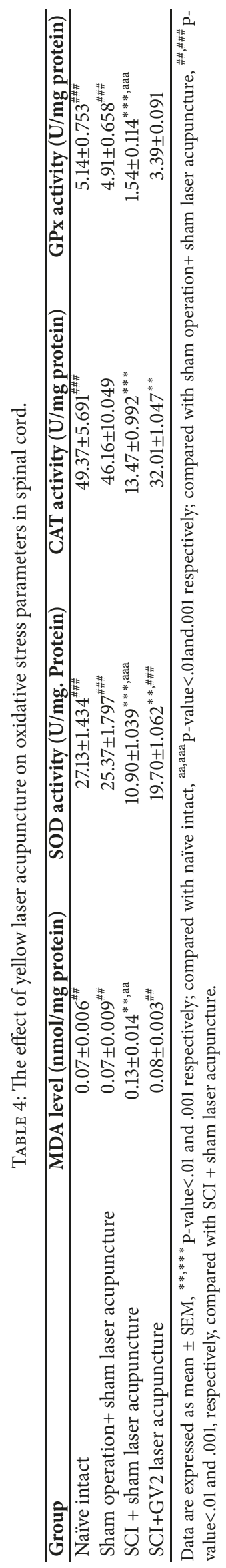




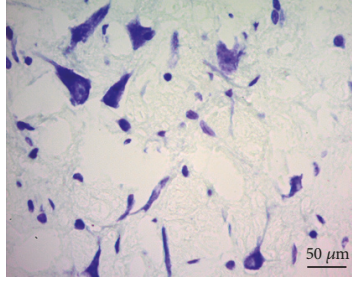

Naïve intact

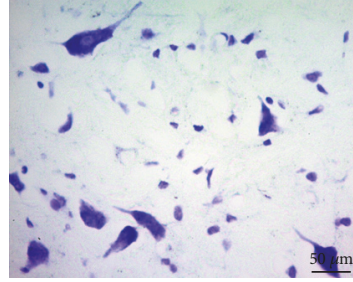

Sham operation+ sham laser acupuncture

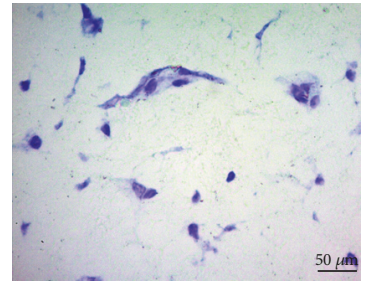

$\mathrm{SCI}+$ sham laser acupuncture

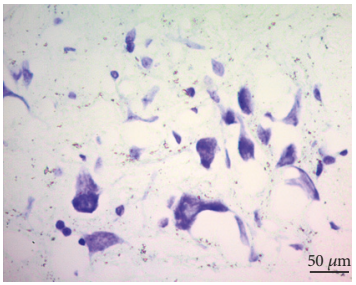

$\mathrm{SCI}+\mathrm{GV} 2$

laser acupuncture

(a)

$\underline{50 \mu \mathrm{m}}$

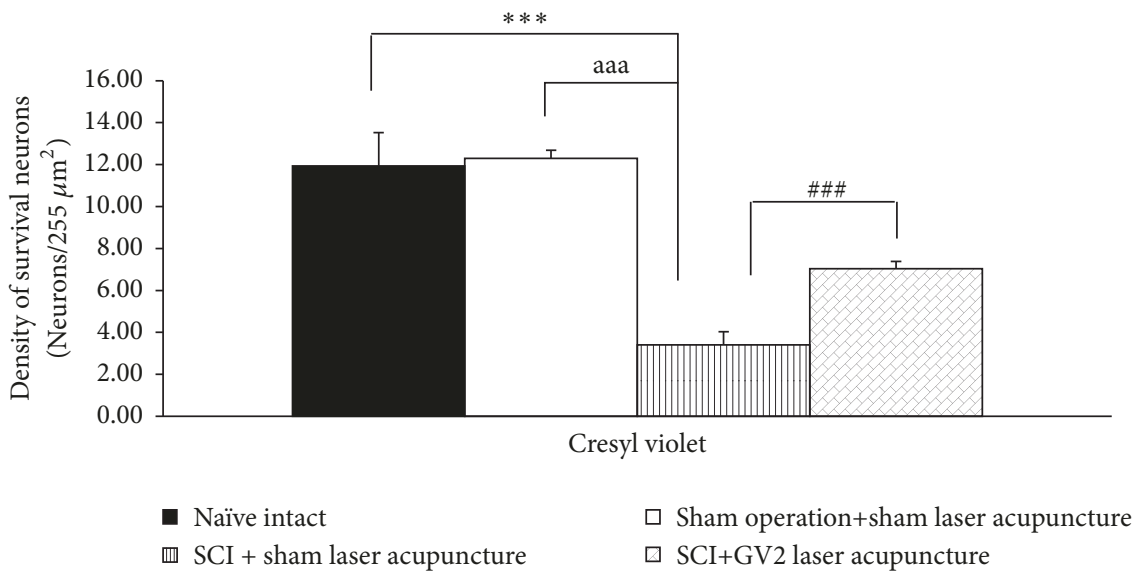

(b)

FIGURE 1: Effect of yellow laser acupuncture on survival neurons (ventral horn) in the spinal cord. Data are expressed as mean \pm SEM, ${ }^{* * *} \mathrm{P}-$ value $<. .001$ compared with naïve intact, ${ }^{\text {aaa }} \mathrm{P}$-value $<.001$ compared with sham operation + sham laser acupuncture, ${ }^{\# \# ~} \mathrm{P}$-value $<.001 \mathrm{compared}$ with SCI + sham laser acupuncture.

were also observed in SCI rats which received GV2 laser acupuncture.

It has been reported that IL-6 is the principal proinflammatory cytokine in SCI [29]. This cytokine plays roles in regulating various steps in inflammatory reactions, such as the activation and infiltration of neutrophils, monocytes, macrophages, and lymphocytes [30]. In addition, among various types of leukocytes mentioned earlier, neutrophil is regarded as the most potent triggers of post-traumatic spinal cord damage. Based on the crucial roles of both IL- 6 and polymorphonuclear leukocyte (neutrophil) density, both parameters were explored in this study. Interestingly, the stimulation of GV2 acupoint by yellow laser decreased polymorphonuclear leukocyte density. Therefore, the increased survival neurons in ventral horn of spinal cord and the improved locomotor activity of SCI rats which received laser stimulation at GV2 might occur partly via the decreased inflammation and via the reduction of polymorphonuclear leukocyte density. It was found that COX-2 activity was also upregulated in SCI rats and this change was mitigated by yellow laser acupuncture at GV2 acupoint. Based on this information, we did suggest that the decrease in inflammation might also occur partly via the reduction of COX- 2 .

The data obtained from this study demonstrated that the SCI rats showed the reduction of the main scavenger enzymes, including SOD and GPx, leading to the excess of superoxide anion and hydrogen peroxide and the attack of polyunsaturated fatty acid (PUFA) in the membranes of various organelles resulting in the elevation of malondialdehyde (MDA) level. These results were in agreement many the previous studies $[31,32]$. GV2 stimulation by yellow laser could attenuate the reduction of SOD and the elevation of MDA level of SCI rats. In addition, it has been reported that oxidative stress can induce inflammatory response which in turn leads to apoptosis and neurological deficit [33]. Therefore, the decreased inflammation in SCI rats might occur not only via the reduction of polymorphonuclear leukocyte density but also via the reduction of oxidative stress. However, the reduction of inflammation can also decrease oxidative stress [34]. Therefore, the reduction of oxidative stress induced by the stimulation induced by the stimulation of GV2 also occurs partly due to the reduction of 


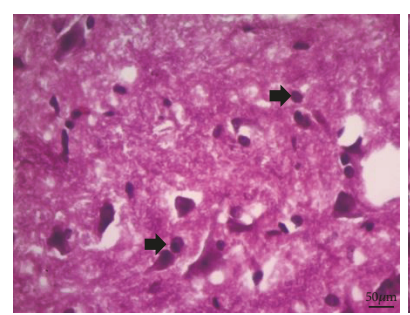

Naïve intact

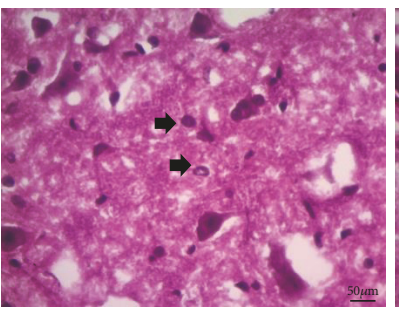

Sham operation+ sham laser acupuncture

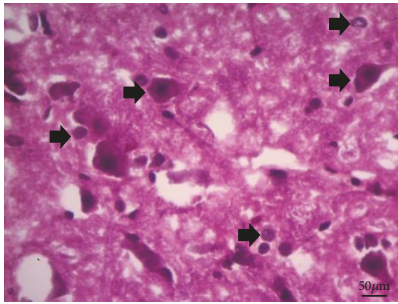

SCI + sham laser acupuncture

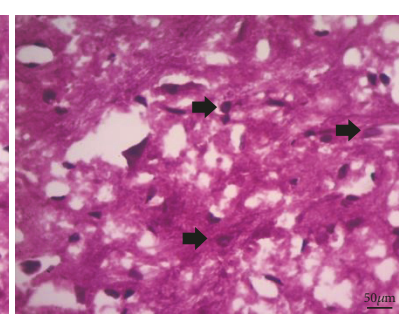

$\mathrm{SCI}+\mathrm{GV} 2$

laser acupuncture

(a)

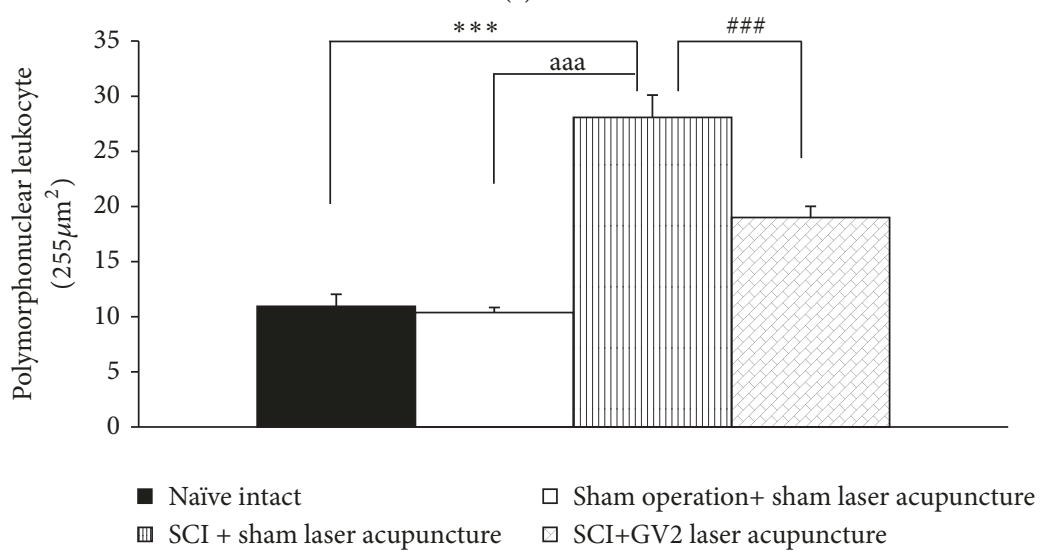

(b)

FIGURE 2: Effect of yellow laser acupuncture on polymorphonuclear leukocyte (ventral horn) in the spinal cord. Data are expressed as mean \pm SEM, ${ }^{* * *} \mathrm{P}$-value $<.001$ compared with naïve intact, ${ }^{\text {aaa }} \mathrm{P}$-value $<.001$ compared with sham operation + sham laser acupuncture, and ${ }^{\# \# \#} \mathrm{P}$ value $<.001$ compared with SCI + sham laser acupuncture.

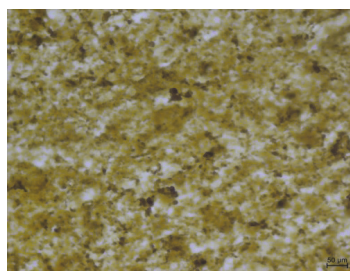

Naïve intact

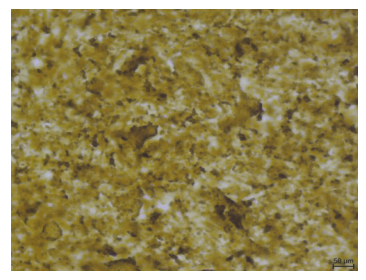

Sham operation+ sham laser acupuncture

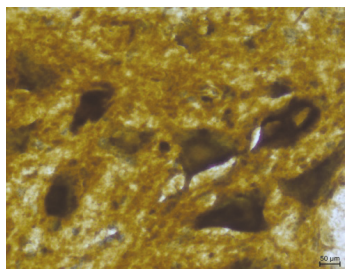

SCI + sham laser acupuncture

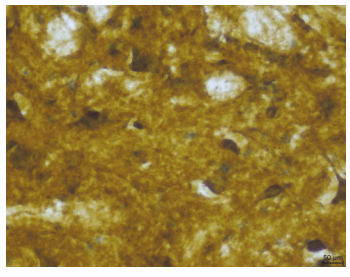

$\mathrm{SCI}+\mathrm{GV} 2$

laser acupuncture

(a)

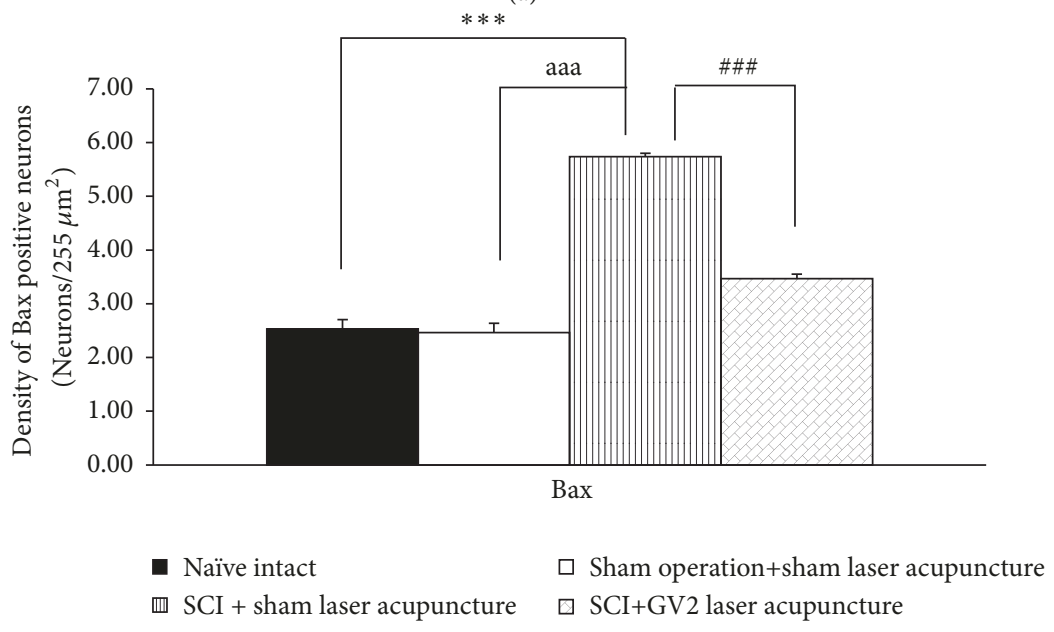

(b)

FiguRE 3: Effect of yellow laser acupuncture on Bax positive stained neurons density in ventral horn of spinal cord injury rats. Data are expressed as mean $\pm \mathrm{SEM},{ }^{* * *} \mathrm{P}$-value $<.001$ compared with Naïve intact ${ }^{\text {aaa }} \mathrm{P}$-value $<.001$ compared with sham operation + sham laser acupuncture, ${ }^{\# \#} \mathrm{P}$-value $<.001$ compared with SCI + sham laser acupuncture. 


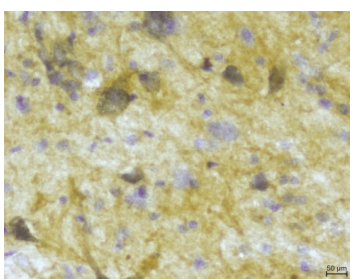

Naïve intact

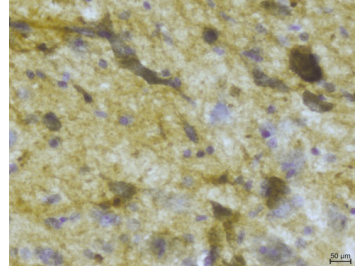

Sham operation + sham laser acupuncture

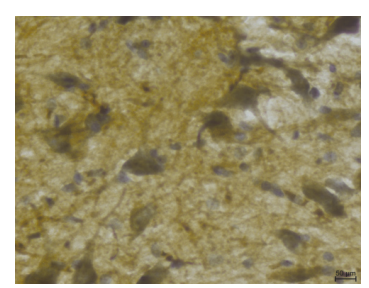

$\mathrm{SCI}+$ sham laser acupuncture

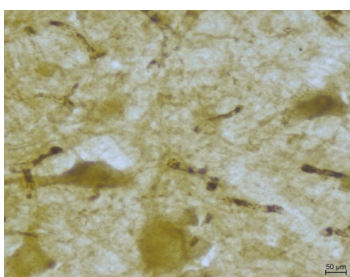

$\mathrm{SCI}+\mathrm{GV} 2$

laser acupuncture

(a)

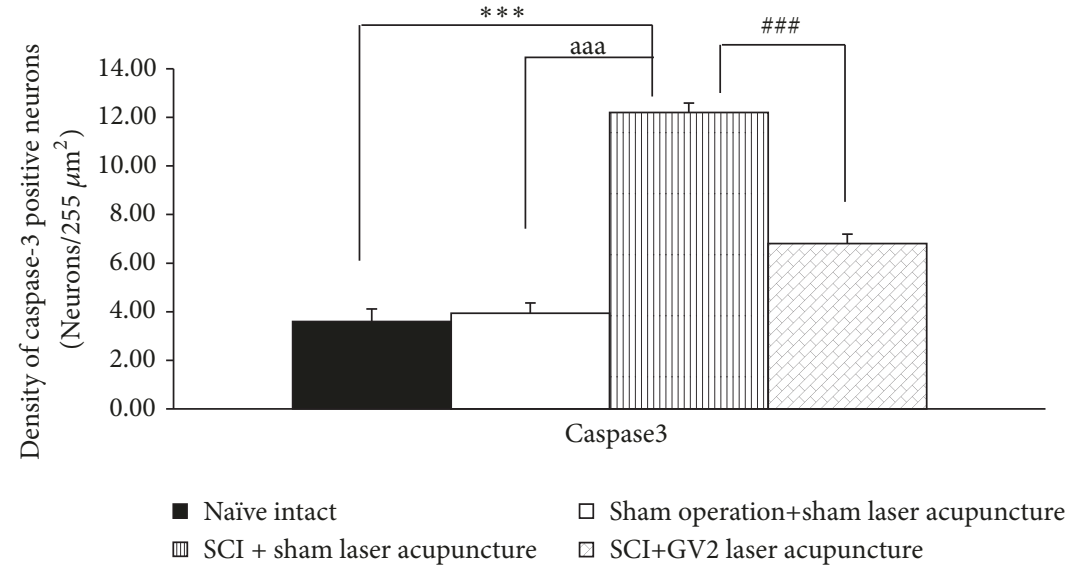

(b)

FIGURE 4: Effect of yellow laser acupuncture on Caspase 3 positive stained neurons density in ventral horn of spinal cord injury rats. Data are expressed as mean $\pm \mathrm{SEM},{ }^{* * *} \mathrm{P}$-value $<.001$ compared with naïve intact, ${ }^{\text {aaa }} \mathrm{P}$-value $<0.001$ compared with sham operation + sham laser acupuncture, and ${ }^{\# \# \#} \mathrm{P}$-value $<.001$ compared with SCI + sham laser acupuncture.

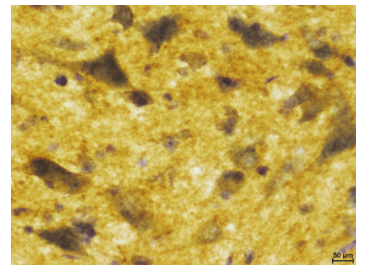

Naïve intact

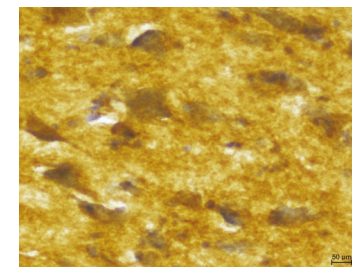

Sham operation + sham laser acupuncture

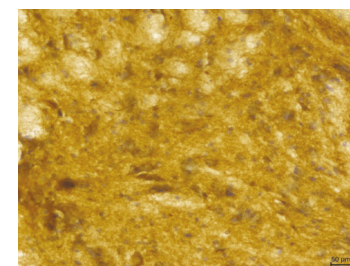

SCI + sham laser acupuncture

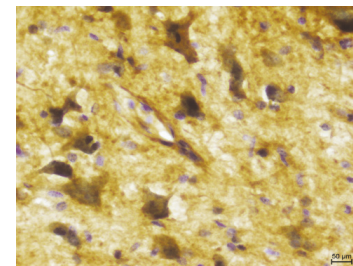

$\mathrm{SCI}+\mathrm{GV} 2$

laser acupuncture

(a)

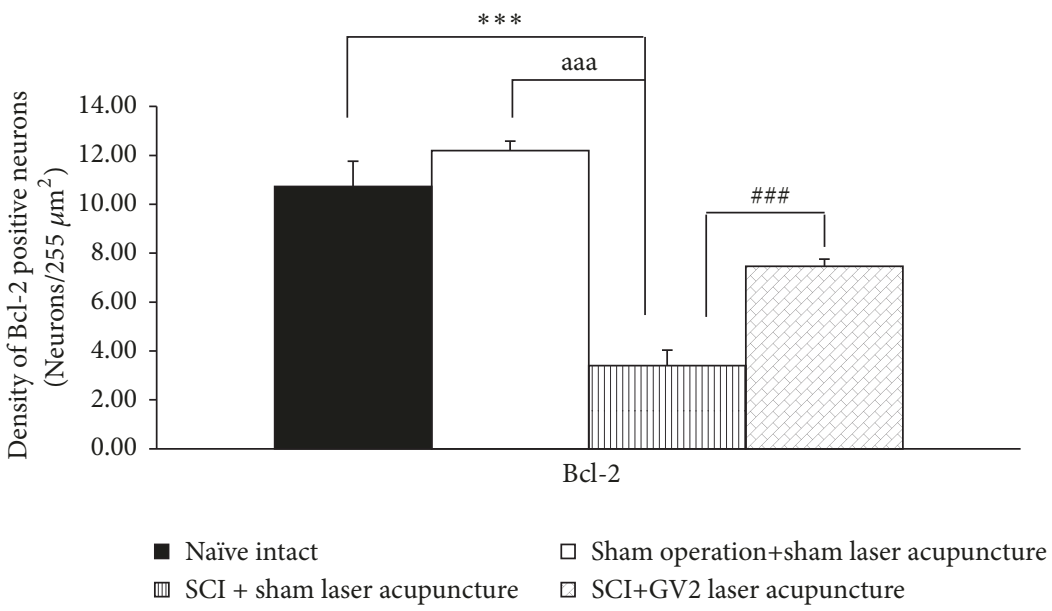

(b)

Figure 5: Effect of yellow laser acupuncture on Bcl-2 positive stained neurons density in ventral horn of spinal cord injury rats. Data are expressed as mean $\pm \mathrm{SEM},{ }^{* * *} \mathrm{P}$-value $<.001$ compared with naïve intact, ${ }^{\text {aaa }} \mathrm{P}$-value $<.001$ compared with sham operation + sham laser acupuncture, and ${ }^{\# \# \#} \mathrm{P}$-value $<.001$, respectively; compared with SCI + sham laser acupuncture. 


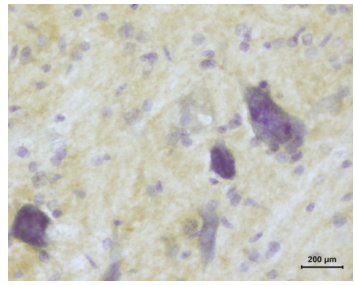

Naïve intact

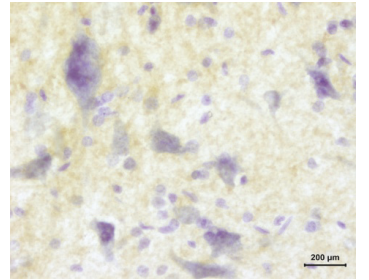

Sham operation+ sham laser acupuncture

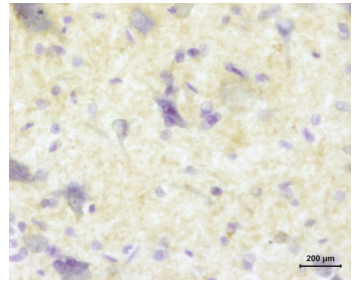

$\mathrm{SCI}+$ sham laser acupuncture

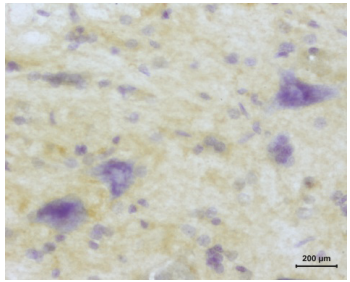

$\mathrm{SCI}+\mathrm{GV} 2$ laser acupuncture

(a)

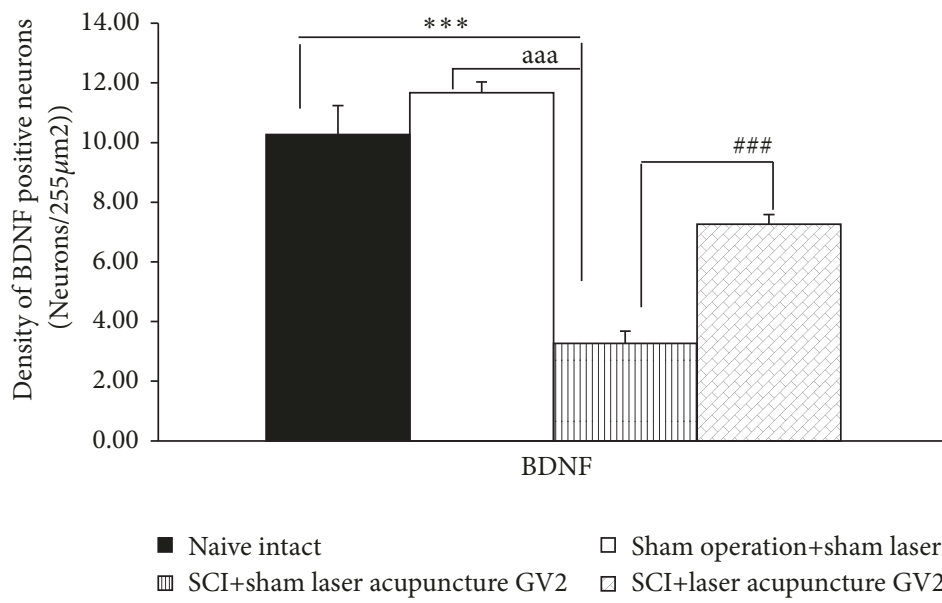

(b)

FIGURE 6: Effect of yellow laser acupuncture on BDNF-positive stained neurons density in ventral horn of spinal cord injury rats. Data are expressed as mean $\pm \mathrm{SEM},{ }^{* * *} \mathrm{P}$-value $<.001$ compared with naïve intact, ${ }^{\text {aaa }} \mathrm{P}$-value $<.001$ compared with sham operation + sham laser acupuncture, and ${ }^{\# \# \#} \mathrm{P}$-value $<.001$, respectively; compared with $\mathrm{SCI}+$ sham laser acupuncture.

inflammation. Interestingly, the stimulation of GV2 by yellow laser could enhance SOD activity. It has been reported that SOD, an important antioxidant enzyme, catalyzes the dismutation of superoxide anion $\left(\mathrm{O}_{2}{ }^{-}\right)$to $\mathrm{H}_{2} \mathrm{O}_{2}$ [35]. Therefore the reduction MDA observed in SCI rats which received GV2 stimulation by yellow laser might occur partly via the increase in SOD which in turn decreased $\mathrm{H}_{2} \mathrm{O}_{2}$ and the attack of free radicals to lipid component of membrane leading to the reduction of neurodegeneration.

Following SCI, apoptosis also occurs and plays a role on the functional disability $[36,37]$. This process is regulated by Bcl-2, Caspase 3, and Bax $[38,39]$. Our data revealed that following SCI, Bax and Caspase 3 positive cells were upregulated whereas Bcl-2 positive cell was downregulated. These data were in agreement with the previous study [39]. Interestingly, our data demonstrated that the stimulation at GV2 acupoint significantly increased the density of Bcl-2 positive cell but decreased the densities of Bax and Caspase- 3 positive cells in ventral horn of the lesion spinal cord.

The current data showed that the density of survival neurons in ventral horn of spinal cord increased. The underlying mechanism is most likely to be associated with the decreased apoptosis (density of Bax-positive cell decreased but density of Bcl-2-positive cell increased). Therefore, the growth factor may contribute a role on this process. However, the growth factor which contributes essential role on the recovery of spinal cord following injury and has gained much attention as therapeutic strategy against spinal cord injury is brain derived growth factor (BDNF) [40]. It can rescue neurons from degenerative atrophy and apoptotic cell death [41, 42]. The current results showed that laser acupuncture at GV2 acupoint significantly enhanced BDNF positive cell in SCI rats. Therefore, the increased survival neuron density together with the decreased apoptosis might be associated with the increase in BDNF which in turn enhanced the survival of neuron and suppressed apoptosis.

Taking all data together, our data suggested that SCI rats which received GV2 stimulation by yellow laser significantly enhanced BDNF producing cells which in turn increased the survival neuron and suppressed apoptosis of neurons in ventral horn of spinal cord. In addition GV2 laser acupuncture also mitigated oxidative stress status and inflammation. These processes also play the roles on the increase neuron density in ventral horn which in turn improved locomotor activity of SCI rats. The elevation of glial cell derived growth factor (GDNF) which plays an important role on the promotion of axonal regeneration and myelination may also involve the improvement of functional outcome of spinal cord following traumatic injury. However, this required further studies.

\section{Conclusion}

In conclusion, the stimulation at GV2 acupoint by yellow laser is the potential novel intervention to improve both 
motor deficit and neurodegeneration after traumatic injury in ventral horn of spinal cord. It should provide health benefit for many disability patients and decrease annual healthcare budget related to the management of disability of traumatic spinal cord injury. However, the clinical trial study is still essential before moving forward for further application.

\section{Data Availability}

The data used to support the findings of this study are available from the corresponding author upon request.

\section{Conflicts of Interest}

The authors declare that they have no conflicts of interest.

\section{Authors' Contributions}

Parichat On-ong-arj collected data and prepared figure and table; Jintanaporn Wattanathorn designed experiment, analyzed data, and prepared whole manuscripts; Supaporn Muchimapura handled training surgery and cord injury induction; Wipawee Thukham-mee prepared data and data presentation

\section{Acknowledgments}

This study was supported by National Research Council of Thailand, Integrative Complementary Alternative Research and Development Center, and Invitation Research of Research Division, Faculty of Medicine, Khon Kaen University, Khon Kaen, Thailand. Since the work submitted here is some part of Ph.D thesis of Parichat On-ong-arj, the authors also would like to thank Graduate School, Khon Kaen University, Khon Kaen, Thailand, for providing the opportunity to publish this work.

\section{References}

[1] G.-Z. Ning, Q. Wu, Y.-L. Li, and S.-Q. Feng, "Epidemiology of traumatic spinal cord injury in Asia: a systematic review," The Journal of Spinal Cord Medicine, vol. 35, no. 4, pp. 229-239, 2012.

[2] World Health Organization, "International Perspectives on Spinal Cord Injury," 2013, http://www.who.int/disabilities/ policies/spinal_cord_injury/en/, Accessed on 1 February 2018.

[3] P. F. Stahel, T. Vanderheiden, and M. A. Finn, "Management strategies for acute spinal cord injury: Current options and future perspectives," Current Opinion in Critical Care, vol. 18, no. 6, pp. 651-660, 2012.

[4] C. A. Oyinbo, "Secondary injury mechanisms in traumatic spinal cord injury: a nugget of this multiply cascade," Acta Neurobiologiae Experimentalis, vol. 71, no. 2, pp. 281-299, 2011.

[5] Q. Yan, J.-W. Ruan, Y. Ding, W.-J. Li, Y. Li, and Y.-S. Zeng, "Electro-acupuncture promotes differentiation of mesenchymal stem cells, regeneration of nerve fibers and partial functional recovery after spinal cord injury," Experimental and Toxicologic Pathology, vol. 63, no. 1-2, pp. 151-156, 2011.

[6] Y.-T. Zhang, H. Jin, J.-H. Wang et al., "Tail nerve electrical stimulation and electro-acupuncture can protect spinal motor neurons and alleviate muscle atrophy after spinal cord transection in rats," Neural Plasticity, vol. 2017, Article ID 7351238, 11 pages, 2017.

[7] X. Wu, A. E. Dmitriev, M. J. Cardoso et al., " $810 \mathrm{~nm}$ wavelength light: An effective therapy for transected or contused rat spinal cord," Lasers in Surgery and Medicine, vol. 41, no. 1, pp. 36-41, 2009.

[8] J. T. Hashmi, Y. Huang, B. Z. Osmani, S. K. Sharma, M. A. Naeser, and M. R. Hamblin, "Role of low-level laser therapy in neurorehabilitation," PMßR : The Journal of Injury, Function, and Rehabilitation, vol. 2, no. 12, pp. S292-S305, 2010.

[9] J. Khongrum and J. Wattanathorn, "Laser acupuncture at HT7 improves the cerebellar disorders in valproic acid-rat model of autism," JAMS Journal of Acupuncture and Meridian Studies, vol. 10, no. 4, pp. 231-239, 2017.

[10] J. Khongrum and J. Wattanathorn, "Laser acupuncture improves behavioral disorders and brain oxidative stress status in the valproic acid rat model of autism," Journal of Acupuncture and Meridian Studies, vol. 8, no. 4, pp. 183-191, 2015.

[11] N. Phunchago, J. Wattanathorn, K. Chaisiwamongkol, S. Muchimapura, and W. Thukham-mee, "Acupuncture reduces memory impairment and oxidative stress and enhances cholinergic function in an animal model of alcoholism," JAMS Journal of Acupuncture and Meridian Studies, vol. 8, no. 1, pp. 23-29, 2015.

[12] C. Sutalangka, J. Wattanathorn, S. Muchimapura, W. ThukhamMee, P. Wannanon, and T. Tong-un, "Laser acupuncture improves memory impairment in an animal model of Alzheimer's disease," Journal of Acupuncture and Meridian Studies, vol. 6, no. 5, pp. 247-251, 2013.

[13] J. C. Bruce, M. A. Oatway, and L. C. Weaver, "Chronic pain after clip-compression injury of the rat spinal cord," Experimental Neurology, vol. 178, no. 1, pp. 33-48, 2002.

[14] D. M. Basso, M. S. Beattie, and J. C. Bresnahan, "A sensitive and reliable locomotor rating scale for open field testing in rats," Journal of Neurotrauma, vol. 12, no. 1, pp. 1-21, 1995.

[15] T. E. P. De Barros Filho and A. E. I. S. Molina, "Analysis of the sensitivity and reproducibility of the Basso, Beattie, Bresnahan (BBB) scale in wistar rats," Clinics, vol. 63, no. 1, pp. 103-108, 2008.

[16] P. Anand, D. C. Mathangi, M. Jeraud, A. Namasivayam, and R. S. Babu, "Behavioral analysis after sciatic nerve compression in albino rats," Annals of Neurosciences, vol. 18, no. 2, pp. 37-43, 2011.

[17] Y. H. O. Yang, Z. Wang, J. Zheng, and R. Wang, "Protective effects of gallic acid against spinal cord injury-induced oxidative stress," Molecular Medicine Reports, vol. 12, no. 2, pp. 3017-3024, 2015.

[18] H. Ohkawa, N. Ohishi, and K. Yagi, "Assay for lipid peroxides in animal tissues by thiobarbituric acid reaction," Analytical Biochemistry, vol. 95, no. 2, pp. 351-358, 1979.

[19] Y. Sun, L. W. Oberley, and Y. Li, "A simple method for clinical assay of superoxide dismutase," Clinical Chemistry, vol. 34, no. 3, pp. 497-500, 1988.

[20] L. Goth, "A simple method for determination of serum catalase activity and revision of reference range," Clinica Chimica Acta, vol. 196, no. 2-3, pp. 143-151, 1991.

[21] J. T. Rotruck, A. L. Pope, H. E. Ganther, A. B. Swanson, D. G. Hafeman, and W. G. Hoekstra, "Selenium: biochemical role as a component of glatathione peroxidase," Science, vol. 179, no. 4073, pp. 588-590, 1973. 
[22] H. Cho, C.-W. Yun, W.-K. Park et al., "Modulation of the activity of pro-inflammatory enzymes, COX-2 and iNOS, by chrysin derivatives," Pharmacological Research, vol. 49, no. 1, pp. 37-43, 2004.

[23] A. McDonough, A. Monterrubio, J. Ariza, and V. MartínezCerdeño, "Calibrated forceps model of spinal cord compression injury," Journal of Visualized Experiments, vol. 2015, no. 98, 2015.

[24] K.-L. Zhou, D.-H. Chen, H.-M. Jin et al., "Effects of calcitriol on experimental spinal cord injury in rats," Spinal Cord, vol. 54, no. 7, pp. 510-516, 2016.

[25] L. Fan, K. Wang, Z. Shi, J. Die, C. Wang, and X. Dang, "Tetramethylpyrazine protects spinal cord and reduces inflammation in a rat model of spinal cord ischemia-reperfusion injury," Journal of Vascular Surgery, vol. 54, no. 1, pp. 192-200, 2011.

[26] M. H. Chen, Q. X. Ren, W. F. Yang et al., "Influences of HIFla on Bax/Bcl-2 and VEGF expressions in rats with spinal cord injury," International Journal of Clinical and Experimental Pathology, vol. 6, no. 11, pp. 2312-2322, 2013.

[27] J. Sun, C. Xie, W. Liu et al., "The effects of simvastatin on hippocampal caspase- 3 and Bcl-2 expression following kainateinduced seizures in rats," International Journal of Molecular Medicine, vol. 30, no. 4, pp. 739-746, 2012.

[28] S. Okada, M. Nakamura, Y. Mikami et al., "Blockade of Interleukin-6 Receptor Suppresses Reactive Astrogliosis and Ameliorates Functional Recovery in Experimental Spinal Cord Injury," Journal of Neuroscience Research, vol. 76, no. 2, pp. 265-276, 2004.

[29] S. Lacroix, L. Chang, S. Rose-John, and M. H. Tuszynski, "Delivery of hyper-interleukin-6 to the injured spinal cord increases neutrophil and macrophage infiltration and inhibits axonal growth," Journal of Comparative Neurology, vol. 454, no. 3, pp. 213-228, 2002.

[30] M. Y. Kaynar, M. Hanci, C. Kuday, A. Belce, K. Gumustas, and E. Kokoglu, "Changes in the activity of antioxidant enzymes (SOD, GPX, CAT) after experimental spinal cord injury," The Tokushima journal of experimental medicine, vol. 41, no. 3-4, pp. 133-136, 1994.

[31] C.-Y. Wang, J.-K. Chen, Y.-T. Wu et al., "Reduction in antioxidant enzyme expression and sustained inflammation enhance tissue damage in the subacute phase of spinal cord contusive injury," Journal of Biomedical Science, vol. 18, no. 13, pp. 1-16, 2011.

[32] T. Genovese, E. Esposito, E. Mazzon et al., "Absence of endogenous interleukin-10 enhances secondary inflammatory process after spinal cord compression injury in mice," Journal of Neurochemistry, vol. 108, no. 6, pp. 1360-1372, 2009.

[33] O. H. Bedreag, A. F. Rogobete, M. Sărăndan et al., "Oxidative stress and antioxidant therapy in traumatic spinal cord injuries," Romanian Journal of Anaesthesia and Intensive Care, vol. 21, no. 2, pp. 123-129, 2014.

[34] O. Ighodaro and O. Akinloye, "First line defence antioxidantssuperoxide dismutase (SOD), catalase (CAT) and glutathione peroxidase (GPX): Their fundamental role in the entire antioxidant defence grid," Alexandria Journal of Medicine, 2017.

[35] L. Zhang, P. Tang, H. Hou et al., "Autophagy reduces neuronal damage and promotes locomotor recovery via inhibition of apoptosis after spinal cord injury in rats," Molecular Neurobiology, vol. 49, no. 1, pp. 276-287, 2014.

[36] X. Chen, X. Chen, X. Huang et al., "Soluble epoxide hydrolase inhibition provides multi-target therapeutic effects in rats after spinal cord injury," Molecular Neurobiology, vol. 53, no. 3, pp. 1565-1578, 2016.
[37] G. M. Cohen, “Caspases: The executioners of apoptosis," Biochemical Journal, vol. 326, part 1, pp. 1-16, 1997.

[38] D. W. Choi, "Neuronal and glial apoptosis after traumatic spinal cord injury," The Journal of Neuroscience, vol. 17, no. 14, pp. 53955406, 1997.

[39] J. Yuan and B. A. Yankner, "Apoptosis in the nervous system," Nature, vol. 407, no. 6805, pp. 802-809, 2000.

[40] A. H. Nagahara and M. H. Tuszynski, "Potential therapeutic uses of BDNF in neurological and psychiatric disorders," Nature Reviews Drug Discovery, vol. 10, no. 3, pp. 209-219, 2011.

[41] N. R. Kobayashi, D.-P. Fan, K. M. Giehl, A. M. Bedard, S. J. Wiegand, and W. Tetzlaff, "BDNF and NT-4/5 prevent atrophy of rat rubrospinal neurons after cervical axotomy, stimulate GAP-43 and T $\alpha 1$-tubulin mRNA expression, and promote axonal regeneration," The Journal of Neuroscience, vol. 17, no. 24, pp. 9583-9595, 1997.

[42] P. Lu, A. Blesch, and M. H. Tuszynski, "Neurotrophism without neurotropism: BDNF promotes survival but not growth of lesioned corticospinal neurons," Journal of Comparative Neurology, vol. 436, no. 4, pp. 456-470, 2001. 


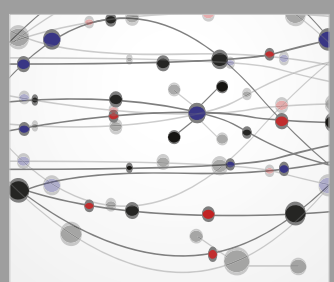

The Scientific World Journal
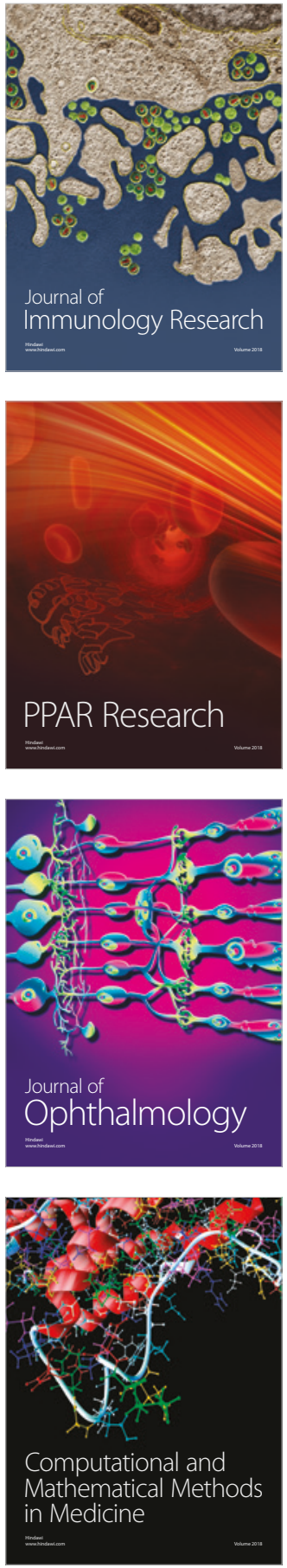

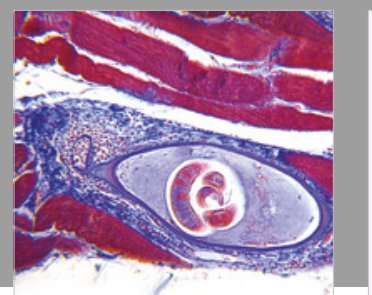

Gastroenterology Research and Practice

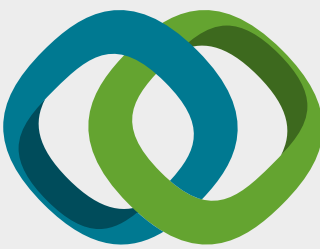

\section{Hindawi}

Submit your manuscripts at

www.hindawi.com
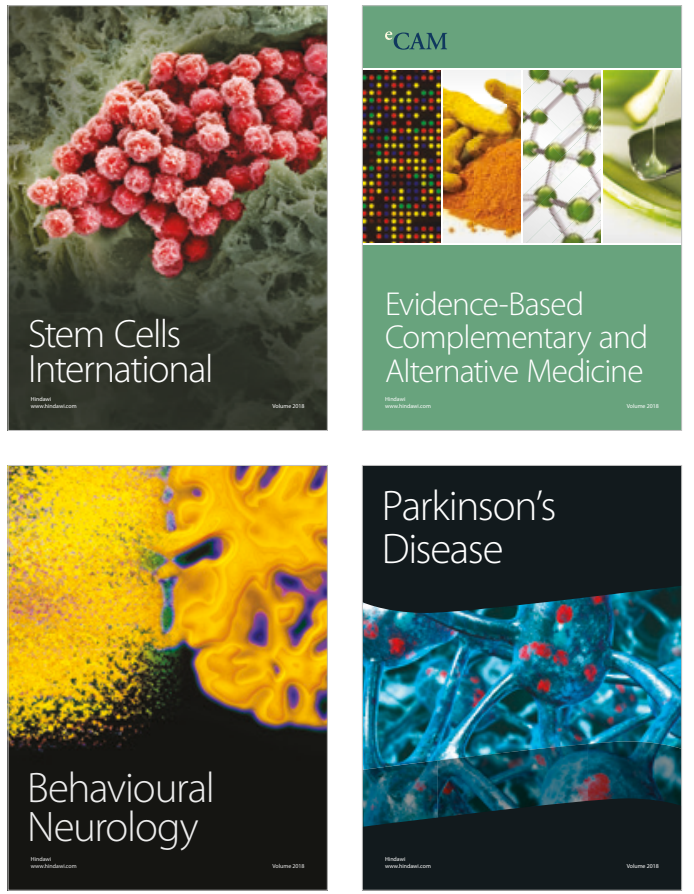

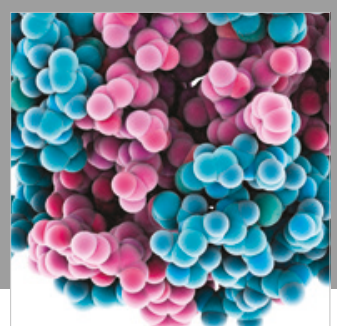

ournal of

Diabetes Research

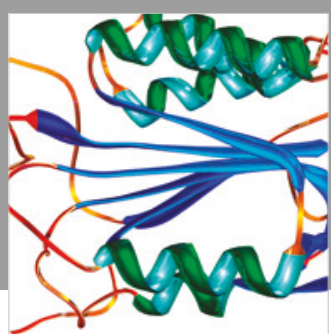

Disease Markers
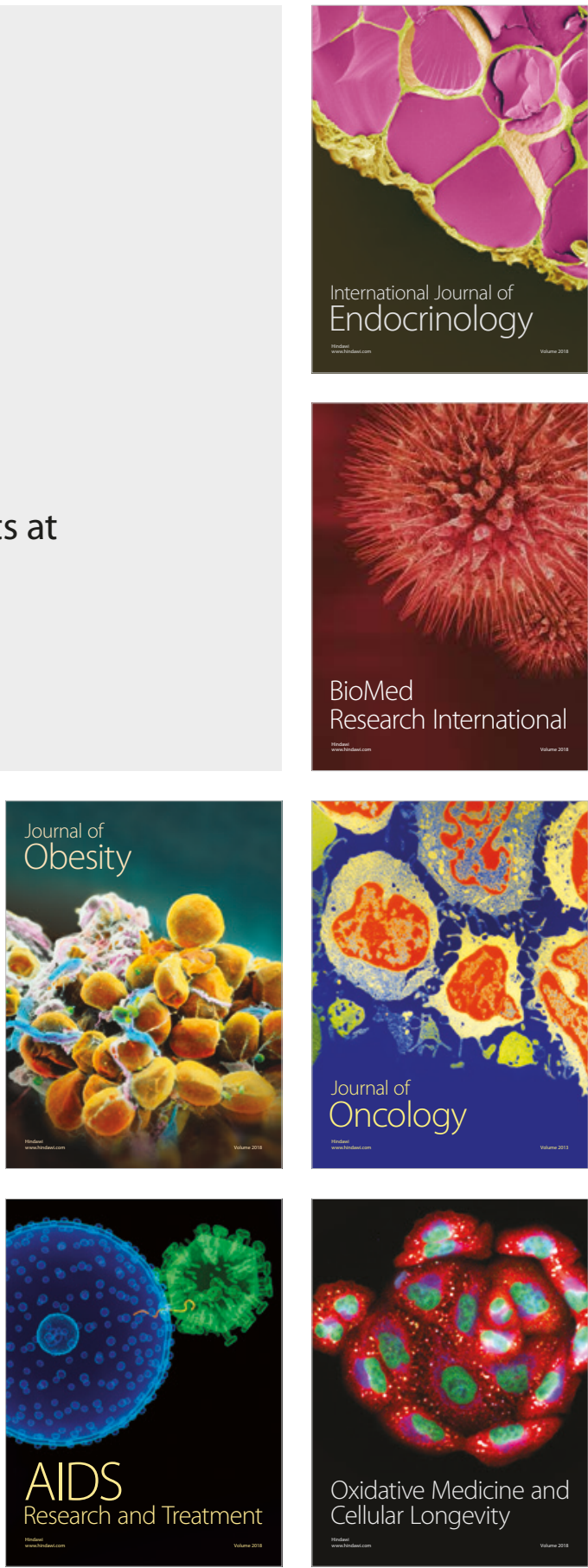